The modulus of elasticity changes by less than $2 \%$ over the entire temperature range investigated. The room temperature modulus using a simple beam is $11.75 \times 10^{6}$ psi.|l This value agrees very well with $11.6 \times 10^{6}$ psi given by Spinner ${ }^{10}$ using a dynamic (resonance) method.

\section{CONCLUSION}

Abrading decreases the strength of glass at these low temperatures in the same way as at higher temperatures. Lowering the test temperature increases the strength, up to a point, of both abraded and unabraded specimens. The breaking stress at $194^{\circ} \mathrm{K}$ and $296^{\circ} \mathrm{K}$ is dependent on the rate of loading but appears to be independent of the rate at $20^{\circ} \mathrm{K}$ and $76^{\circ} \mathrm{K}$. Contact with liquid nitrogen and hydrogen does not appear to affect adversely the breaking stress.

The fatigue or endurance limit at $76^{\circ} \mathrm{K}$ appears to be greater than 9000 psi. However, since some of the specimens withstood loads of 10000 psi for several

II C. J. Parker (private communication).

10 Sam Spinner, J. Am. Ceram. Soc. 39, 113 (1956). hours and then fractured, the existence of some fatigue is indicated. There appears to be some agreement between these data and those of Vonnegut and Glathart. The agreement is good at $76^{\circ} \mathrm{K}$ and $296^{\circ} \mathrm{K}$ but deviates markedly at $194^{\circ} \mathrm{K}$. This may result from the method used in plotting the data or from the fact that the specimens were tested in contact with acetone and dry ice in one case and air in the other.

The results of these tests show that the strength of glass is essentially the same at $20^{\circ} \mathrm{K}$ as it is at $76^{\circ} \mathrm{K}$. In addition, at these low temperatures glass exhibits very little or no fatigue, and therefore much higher design stresses can be used for glass in cryogenic apparatus.

\section{ACKNOWLEDGMENTS}

The authors wish to thank E. B. Shand, C. J. Parker, and Dr. W. W. Shaver of the Corning Glass Works for their assistance in furnishing the test specimens and for their aid in data interpretation. In addition, we wish to express our appreciation to $\mathrm{E}$. $\mathrm{H}$. Brown of the National Bureau of Standards for his help in this work.

\title{
Infrared Emissivities and Absorptivities of Gases*
}

\author{
S. S. Penner and A. Thomson \\ Daniel and Florence Guggenheim Jet Propulsion Center, California Institute of Technology, Pasadena, California
}

(Received September 18, 1956)

\begin{abstract}
Approximate analytic expressions are obtained for the "effective band widths" and emissivities of diatomic molecules at elevated pressures.

The absorptivities of molecular vibration-rotation bands are evaluated for radiation emitted by similar molecular vibration-rotation bands and for radiation emitted by blackbodies. The results obtained for the absorptivity calculations compare favorably with available empirical correlations for $\mathrm{H}_{2} \mathrm{O}$.

The postulated model does not apply to $\mathrm{CO}_{2}$ and, therefore, no satisfactory theoretical formula has been derived for the absorptivities of this molecule. The appendix (by A. Thomson) is devoted to a critical examination of the limits of validity of the effective band-width concept for different molecules.
\end{abstract}

\section{INTRODUCTION}

$I^{N}$ NFRARED emissivity calculations for diatomic gases were carried out some years ago for (a) elevated pressures where extensive overlapping occurs between the rotational lines of the vibration-rotation bands ${ }^{1}$ and (b) low pressures and low optical densities where the rotational lines may be considered to be completely separated. ${ }^{2}$

In the following Sec. II we consider a formal addition to the ideas published in earlier papers and obtain approximate analytic expressions for the effective band

* Supported by the U. S. Office of Naval Research under Contract Nonr-220(03), NR 015401.

1 (a) S. S. Penner, J. Appl. Phys. 21, 685 (1950); (b) S. S. Penner, J. Appl. Mech. 18, 53 (1951); (c) S. S. Penner and D. Weber, J. Appl. Phys. 22, 1164 (1951).

2 (a) Penner, Ostrander, and Tsien, J. Appl. Phys. 23, 256 (1952); (b) S. S. Penner, J. Appl. Phys. 23, 825 (1952). widths and emissivities of diatomic molecules in the special case where the occurrence of band heads may be ignored.

Section III is devoted to a quantitative discussion of the absorptivity of molecular vibration-rotation bands with overlapping rotational lines for radiation emitted either from molecular vibration-rotation bands or from a blackbody. Our theoretical results are compared with an empirical relation ${ }^{3}$ and are shown to be useful for correlating experimental data for water vapor for optical densities which are sufficiently high to justify the assumption that the rotational fine structure has been completely smeared out.

${ }^{3}$ H. C. Hottel and R. B. Egbert, Trans. Am. Inst. Chem. Eng. 38, 531 (1942); H. C. Hottel and H. G. Mangelsdorf, Trans. Am. Inst. Chem. Eng. 31, 517 (1935). W. H. McAdams, Heat Trans mission (McGraw-Hill Book Company, Inc., New York, 1954), third edition, Chap. III by H. C. Hottel. 


\section{APPROXIMATE ANALYTIC EXPRESSION FOR EFFECTIVE BAND WIDTHS AND EMISSIVITIES OF DIATOMIC MOLECULES ${ }^{4}$}

The procedure for calculating effective band widths was described in an earlier publication. ${ }^{1}$ We use the same physical ideas but obtain analytic expressions which are useful provided the formation of band heads can be ignored. In the event that band heads are important for the range of optical densities and temperatures considered, it is necessary to revert to the original numerical method of calculation. ${ }^{1}$

For diatomic molecules without $Q$ branch, the intensity distribution in a vibration-rotation band is dominated, ${ }^{2}$ for large $j$, by the term

$$
j \exp (-\epsilon(n, j) / k T),
$$

where we refer to the transition $j \rightarrow j-1$. $\dagger$ Here $j$ and $n$ denote, respectively, the rotational and vibrational quantum numbers of the initial state, $\epsilon(n, j)$ is the corresponding energy, $k$ equals the Boltzmann constant, and $T$ is the absolute temperature. In the notation used by Mayer and Mayer, ${ }^{5} \epsilon(n, j) / k T$ may be shown to be given by the expression

$$
\begin{aligned}
\epsilon(n, j) / k T & =u_{e}\left(n+\frac{1}{2}\right)\left[1-x_{e}\left(n+\frac{1}{2}\right)\right] \\
& +\gamma u_{e} j(j+1)\left[1-\left(n+\frac{1}{2}\right) \delta-4 \gamma^{2} j(j+1)\right] .
\end{aligned}
$$

The value of $j=j^{*}$ for which $j \exp [-\epsilon(n, j) / k T]$ attains a maximum is, approximately,

$$
j^{*} \simeq\left(2 \gamma u_{e}\right)^{-\frac{1}{2}},
$$

where $4 \gamma^{2} j(j+1)$, which accounts for band-head formation, and $\left(n+\frac{1}{2}\right) \delta$ have been assumed to be negligibly small. More accurate results may be obtained by replacing $\gamma u_{e}$ everywhere by $\gamma u_{e}\left[1-\left(n+\frac{1}{2}\right) \delta\right]$. This correction causes a slow variation of band width with vibrational quantum number. The maximum value of $j \equiv j_{\max }$, which limits the effective band width, has been defined ${ }^{1}$ by the relation

$$
\begin{aligned}
j_{\text {max }} \exp \left[-\epsilon\left(n, j_{\text {max }}\right) / k T\right] & =10^{-3} j^{*} \exp \left[-\epsilon\left(n, j^{*}\right) / k T\right] .
\end{aligned}
$$

Using Eqs. (1) and (2), it is readily shown that Eq. (3) may be rewritten as

$$
\ln \left(\frac{j_{\max }}{j^{*}}\right)^{2}-\left(\frac{j_{\max }}{j^{*}}\right)^{2}+14.8=0 .
$$

Equation (4) may be solved by an iteration procedure. The result is

$$
j_{\max } \simeq(17.69)^{\frac{1}{2}}\left(j^{*}\right)=\left(8.84 / \gamma u_{e}\right)^{\frac{1}{2}} .
$$

\footnotetext{
4 Calculations similar to those described in this Sec. II were first proposed in 1951 by H. S. Tsien.

$\dagger$ Our calculations refer to the $R$ branch; a similar analysis applies to the $P$ branch.

J. E. Mayer and M. G. Mayer, Statistical Mechanics (John Wiley and Sons, Inc., New York, 1940), Chap. 7.
}

TABLE I, Effective band widths of the fundamental vibrationrotation bands of $\mathrm{CO}$ and $\mathrm{HCl}$ computed from $\mathrm{Eq}$. (7). The values given in parentheses were obtained by numerical calculations. ${ }^{1}$

\begin{tabular}{rcc}
\hline $\begin{array}{c}\mathrm{T} \\
\left({ }^{\circ} \mathrm{K}\right)\end{array}$ & $\begin{array}{c}\Delta \omega_{P}(\mathrm{CO}) \\
\left(\mathrm{cm}^{-1}\right)\end{array}$ & $\begin{array}{c}\Delta \omega_{P}(\mathrm{HCl}) \\
\left(\mathrm{cm}^{-1}\right)\end{array}$ \\
\hline 300 & $239(214)$ & $560(535)$ \\
500 & $308(274)$ & $724(691)$ \\
1000 & $436(379)$ & $1022(945)$ \\
1500 & $535(465)$ & $1250(1178)$ \\
2000 & $617(535)$ & $1450(1314)$ \\
3000 & $756(630)$ & $1772(1665)$ \\
\hline
\end{tabular}

The effective band width of the fundamental vibrationrotation band is then given by the expression

$$
\begin{aligned}
\Delta \omega_{F}=\frac{1}{h c}\left\{\left[\epsilon\left(1, j_{\max }\right)-\right.\right. & \left.\epsilon\left(0, j_{\max }-1\right)\right] \\
& \left.-\left[\epsilon\left(1, j_{\max }-1\right)-\epsilon\left(0, j_{\max }\right)\right]\right\}
\end{aligned}
$$

with a similar relation holding for the harmonic and overtone bands. Using Eq. (1), it is found that, for large $j_{\max }, \mathrm{Eq}$. (6) reduces to

$$
\Delta \omega_{F}=4 \gamma u_{e} k T j_{\max } / h c=11.9\left(\gamma u_{e}\right)^{\frac{1}{k}} k T / h c .
$$

Reference to Eqs. (1) and (6) shows that, for small $\delta$, the band widths of the harmonics and overtones equal $\Delta \omega_{F}$.

Representative values of $\Delta \omega_{F}$, computed from Eq. (7) for $\mathrm{CO}$ and $\mathrm{HCl}$, are summarized in Table I. The values in parentheses are the results obtained previously $^{1}$ by numerical calculations with proper allowance for the occurrence of band heads. Reference to Table I shows that Eq. (7) represents the effective band widths of the fundamental vibration-rotation bands with adequate precision, at least up to temperatures of $3000^{\circ} \mathrm{K}$.

The engineering emissivity $E$ has been expressed ${ }^{1}$ by the relation

$$
\begin{aligned}
E & =\left[1-\exp \left(-\alpha_{F} X / \Delta \omega_{F}\right)\right] \int_{\Delta \omega_{F}} R^{0}(\omega) d \omega / \sigma T^{4} \\
& +\left[1-\exp \left(-\alpha_{0} X / \Delta \omega_{0}\right)\right] \int_{\Delta \omega_{0}} R^{0}(\omega) d \omega / \sigma T^{4}+\cdots,
\end{aligned}
$$

where $\alpha_{F}$ and $\alpha_{0}$ represent, respectively, the integrated absorption for the fundamental and first overtone (in $\mathrm{cm}^{-2}$ atmos $\left.{ }^{-1}\right), R^{0}(\omega)$ is the Planck radiation function (in erg $\mathrm{cm}^{-1} \mathrm{sec}^{-1}$ ), $\sigma$ is the Stefan-Boltzmann constant (in erg cm-2 $\sec ^{-1}{ }^{\circ} \mathrm{K}^{-4}$ ) and $X$ is the optical density (in $\mathrm{cm}$ atmos). With $\Delta \omega_{F}$ and $\Delta \omega_{0}$ given by Eq. (7), Eq. (8) may be used as an explicit representation of the engineering emissivity $E$. 


\section{ABSORPTIVITY OF MOLECULAR VIBRATION- ROTATION BANDS FOR RADIATION EMITTED BY MOLECULAR VIBRATION-ROTATION BANDS}

The following analysis utilizes the concept of an average absorption coefficient for an entire vibrationrotation band. For this reason, the results are applicable only at elevated pressures and moderate or large optical densities where extensive overlapping occurs between rotational lines.

Consider two isothermal parallel layers of the same gas at temperatures $T_{G_{1}}$ and $T_{G 2}$ with optical densities (in $\mathrm{cm}$ atmos) $X_{1}=P_{1} L_{1}$ and $X_{2}=P_{2} L_{2}$, respectively. Here $P_{1}$ and $P_{2}$ denote the partial pressures of absorber and emitter, respectively, whereas $L_{1}$ and $L_{2}$ represent the corresponding geometric path lengths throughout which the gas molecules are distributed.
The total energy emitted by region 2 into an infinitesimal solid angle $\Delta \Omega$ which contains the normal to the interface, in unit time through unit area at the interface between regions 1 and 2 , is

$\Delta I_{2 \rightarrow 1}=\Delta \Omega \int_{0}^{\infty}\left\{1-\exp \left[-k\left(\omega, T_{G 2}\right) X_{2}\right]\right\} R\left(\omega, T_{G 2}\right) d \omega,(9)$

where $k\left(\omega, T_{G 2}\right)$ is the absorption coefficient (in $\mathrm{cm}^{-1}$ atmos $\left.{ }^{-1}\right)$ in region 2 at the wave number $\omega$ and temperature $T_{G 2}$ and $R\left(\omega, T_{G 2}\right)$ equals the Planck radiation function evaluated at the wave number $\omega$ and temperature $T_{G 2}$. The fraction of the energy $\Delta I_{2 \rightarrow 1}$ which is absorbed in region 1 , i.e., the absorptivity $\alpha(2 \rightarrow 1)$ of the gases in region 1 for radiation emitted from region 2 , is

$$
\alpha(2 \rightarrow 1)=\frac{\int_{0}^{\infty}\left\{1-\exp \left[-k\left(\omega, T_{G 1}\right) X_{1}\right]\right\}\left\{1-\exp \left[-k\left(\omega, T_{G 2}\right) X_{2}\right]\right\} R\left(\omega, T_{G 2}\right) d \omega}{\int_{0}^{\infty}\left\{1-\exp \left[-k\left(\omega, T_{G 2}\right) X_{2}\right]\right\} R\left(\omega, T_{G 2}\right) d \omega} .
$$

We now assume ${ }^{1}$ that the $i$ th vibration-rotation band may be described by an average absorption coefficient $\bar{k}_{i}(T)$ over the effective band width $\Delta \omega_{i}(T)$ of the band whose center lies at $\bar{\omega}_{i}$. It has been shown previously ${ }^{1}$ that, for the calculation of over-all emissivities [i.e., for evaluation of the denominator of Eq. (10)],

$$
\bar{k}_{i}(T)=\left(1 / \Delta \omega_{i}\right) \int k(\omega, T) d \omega
$$

represents a good approximation for vibration-rotation bands at pressures which are sufficiently high to smear out the rotational fine structure in such a way that the spectral absorption coefficient $k(\omega, T)$ is no longer a rapidly varying function of $\omega$. The integral in Eq. (11) extends over the $i$ th vibration-rotation band. Exact evaluation of the numerator of Eq. (10), using reasonable representations of $k\left(\omega, T_{g_{1}}\right)$, shows again that, for moderate values of $T_{G 2} / T_{G 1}$, Eq. (11) is a good approximation.

Introduction of average absorption coefficients for each vibration-rotation band in the numerator of $\mathrm{Eq}$. (10) reduces the relation to

$$
\alpha(2 \rightarrow 1)=\sum_{i} \frac{\left\{1-\exp \left[-\bar{k}_{i}\left(T_{G 1}\right) X_{1}\right]\right\}\left\{1-\exp \left[-\bar{k}_{i}\left(T_{G 2}\right) X_{2}\right]\right\} R\left(\bar{\omega}_{i}, T_{G 2}\right) \Delta \omega^{*}{ }_{i}}{\int_{0}^{\infty}\left\{1-\exp \left[-k\left(\omega, T_{G 2}\right) X_{2}\right]\right\} R\left(\omega, T_{G 2}\right) d \omega}
$$

where the approximation has been made of using the value of the Planck function at temperature $T_{G 2}$ evaluated at the band center for each vibration-rotation band. The quantity $\Delta \omega^{*}{ }_{i}$ in Eq. (12) denotes the effective width of the narrower vibration-rotation band since no absorption occurs outside the limits of $\Delta \omega^{*}{ }_{i}$.

‡ The analysis presented in this Sec. III applies to infinitely narrow beams of light passing in a direction normal to two parallel isothermal layers with optical depths $X_{1}$ and $X_{2}$. Alternately, we may consider concentric hemispherical shells with radii $X_{2}$ and $X_{1}+X_{2}$ and collimated beams of light directed from the center of curvature. If $X_{1}+X_{2}$ is the radius of the outer gas layer, $X_{2}$ represents the radius of the inner gas layer, and we let $X_{2}$ approach zero and replace the gas layer by a blackbody, then we obtain the geometric configuration which is customarily employed for the determination of hemispherical absorptivities.
Thus

and

$$
\Delta \omega_{i}^{*}=\Delta \omega_{i}\left(T_{G 2}\right) \text { for } \quad T_{G 2} \leqslant T_{a 1}
$$

$$
\Delta \omega_{i}^{*}=\Delta \omega_{i}\left(T_{G 1}\right) \text { for } \quad T_{G 1} \leqslant T_{G 2}
$$

From Eq. (11) and the relation $\int k(\omega, T) d \omega \sim T^{-1}$ it is apparent that

$$
\frac{\bar{k}_{i}\left(T_{G 1}\right)}{\bar{k}_{i}\left(T_{G 2}\right)}=\frac{T_{G 2}}{T_{G 1}} \frac{\Delta \omega_{i}\left(T_{G 2}\right)}{\Delta \omega_{i}\left(T_{G 1}\right)} .
$$

The first factor in Eq. (13) arises because the number density of absorbers is inversely proportional to the temperature.

The temperature dependence of $\Delta \omega_{i}$ may be determined from Eq. (7). Since $\gamma$ is independent of temper- 
ature and $u_{e}$ is given by the relation

$$
u_{e}=h c \omega_{e} / k T \text {, }
$$

it follows that Eq. (7) becomes

$$
\Delta \omega_{i}=11.9\left(\gamma k \omega_{e} / h c\right)^{\frac{1}{2}} T^{\frac{1}{2}},
$$

whence

$$
\frac{\bar{k}_{i}\left(T_{G 1}\right)}{\bar{k}_{i}\left(T_{G 2}\right)}=\left(\frac{T_{G 2}}{T_{G 1}}\right)^{\prime} .
$$

In view of Eq. (15) we find that

$\Delta \omega^{*}{ }_{i}(T)=\Delta \omega_{i}\left(T_{G 1}\right)=\left(\frac{T_{G 1}}{T_{G 2}}\right)^{\frac{1}{2}} \Delta \omega_{i}\left(T_{G 2}\right)$

and

$$
\text { for } T_{G 2} \geqslant T_{G 1}
$$

$$
\Delta \omega^{*}{ }_{i}(T)=\Delta \omega_{i}\left(T_{G 2}\right) \text { for } \quad T_{G 1} \geqslant T_{G 2} .
$$

Hence Eq. (12) becomes

$\alpha(2 \rightarrow 1)=\sum_{i}\left(\frac{T_{G 1}}{T_{G 2}}\right)^{\frac{1}{2}} \frac{\left\{1-\exp \left[-\bar{k}_{i}\left(T_{G 2}\right) X_{1}\left(T_{G 2} / T_{G 1}\right)^{\frac{3}{2}}\right]\right\}\left\{1-\exp \left[-\bar{k}_{i}\left(T_{G 2}\right) X_{2}\right]\right\} R\left(\bar{\omega}_{i}, T_{G 2}\right) \Delta \omega_{i}\left(T_{G 2}\right)}{\int_{0}^{\infty}\left\{1-\exp \left[-k\left(\omega, T_{G 2}\right) X_{2}\right]\right\} R\left(\omega, T_{G 2}\right) d \omega}$

and

$$
\alpha(2 \rightarrow 1)=\sum_{i} \frac{\left\{1-\exp \left[-\bar{k}_{i}\left(T_{G 2}\right) X_{1}\left(T_{G 2} / T_{G 1}\right)^{\left.\frac{1}{2}\right]}\right]\left\{1-\exp \left[-\bar{k}_{i}\left(T_{G 2}\right) X_{2}\right]\right\} R\left(\bar{\omega}_{i}, T_{G 2}\right) \Delta \omega_{i}\left(T_{G 2}\right)\right.}{\int_{0}^{\infty}\left\{1-\exp \left[-k\left(\omega, T_{G 2}\right) X_{2}\right]\right\} R\left(\omega, T_{G 2}\right) d \omega} \text { for } T_{G 1} \geqslant T_{G 2}
$$

It is now convenient to transform the summation over individual vibration-rotation bands back to an integration over wave numbers. Thus

$\alpha(2 \rightarrow 1)=\left(\frac{T_{G 1}}{T_{G 2}}\right)^{\frac{1}{2}} \frac{\int_{0}^{\infty}\left\{1-\exp \left[-k\left(\omega, T_{G 2}\right) X_{1}\left(T_{G 2} / T_{G 1}\right)^{\frac{2}{2}}\right]\right\}\left\{1-\exp \left[-k\left(\omega, T_{G 2}\right) X_{2}\right]\right\} R\left(\omega, T_{G 2}\right) d \omega}{\int_{0}^{\infty}\left\{1-\exp \left[-k\left(\omega, T_{G 2}\right) X_{2}\right]\right\} R\left(\omega, T_{G 2}\right) d \omega}$

for $T_{G 1} \leqslant T_{G 2}$;

$$
\alpha(2 \rightarrow 1)=\frac{\int_{0}^{\infty}\left\{1-\exp \left[-k\left(\omega, T_{G 2}\right) X_{1}\left(T_{G 2} / T_{G 1}\right)^{2}\right]\right\}\left\{1-\exp \left[-k\left(\omega, T_{G 2}\right) X_{2}\right]\right\} R\left(\omega, T_{G 2}\right) d \omega}{\int_{0}^{\infty}\left\{1-\exp \left[-k\left(\omega, T_{G 2}\right) X_{2}\right]\right\} R\left(\omega, T_{G 2}\right) d \omega} \text { for } T_{G 1} \geqslant T_{G 2} .
$$

In terms of tabulated isothermal hemispherical emissivities $E(T, X), \mathrm{Eq}$. (21) may be rewritten in the form

$$
\alpha(2 \rightarrow 1)=\left\{\begin{array}{c}
\left(\frac{T_{G 1}}{T_{G 2}}\right)^{\frac{1}{2}}\left\{1-\frac{E\left[T_{G 2}, X_{2}+X_{1}\left(T_{G 2} / T_{G 1}\right)^{\frac{1}{2}}\right]-E\left[T_{G 2}, X_{1}\left(T_{G 2} / T_{G 1}\right)^{\frac{2}{2}}\right]}{E\left(T_{G 2}, X_{2}\right)} \text { for } T_{G 1} \leqslant T_{G 2},\right\} \\
\left\{1-\frac{E\left[T_{G 2}, X_{2}+X_{1}\left(T_{G 2} / T_{G 1}\right)^{2}\right]-E\left[T_{G 2}, X_{1}\left(T_{G 2} / T_{G 1}\right)^{2}\right]}{E\left(T_{G 2}, X_{2}\right)} \text { for } T_{G 1} \geqslant T_{G 2}\right\}
\end{array}\right.
$$

Equation (22) represents the desired relation for the hemispherical absorptivity at a temperature $T_{G 1}$ of a gas characterized by vibration-rotation bands for radiation emitted by the same gas at a temperature $T_{a 2}$.

\section{A. Absorptivities of Water Vapor for Blackbody Radiation}

Using measured values for the absorptivity of water vapor for blackbody radiation, Hottel ${ }^{3}$ obtained the following empirical formula for the absorptivity

$$
\alpha_{H}=\left(\frac{T_{g}}{T_{s}}\right)^{0.45} E\left[T_{s}, X\left(T_{s} / T_{g}\right)\right] .
$$

Here $\alpha_{H}$ is the hemispherical absorptivity measured by Hottel for a layer of water vapor at temperature $T_{\sigma}$ and mean optical density $X$ for radiation emitted by a blackbody at temperature $T_{s}$, and $E\left[T_{s}, X\left(T_{s} / T_{g}\right)\right]$ is the tabulated isothermal hemispherical emissivity cor- 


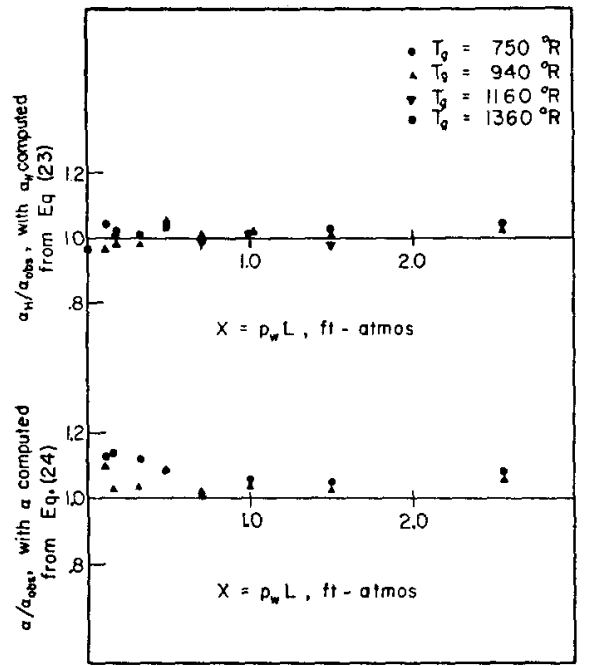

FIG. 1. The ratios $\alpha_{H} / \alpha_{\text {obs }}$ and $\alpha / \alpha_{o b s}$ for blackbody radiation at a temperature $T_{s}=1160^{\circ} \mathrm{R}$.

responding to the temperature $T_{s}$ and an optical density $X\left(T_{s} / T_{g}\right)$. For blackbody radiation, Eq. (22) reduces to the expression (noting that $E\left(T, X_{2}\right)=1$ and that the first of the relations given in Eq. (22) applies now for $T_{G 2} \leqslant T_{G 1}$ as well as for $T_{G 2} \geqslant T_{G 1}$ )

$$
\alpha=\left(\frac{T_{g}}{T_{s}}\right)^{\frac{1}{2}} E\left[T_{s}, X\left(T_{s} / T_{\theta}\right)^{\frac{3}{2}}\right]
$$

Here $T_{G 2}$ has been replaced by $T_{s}, T_{g 1}$ by $T_{g}$ and $X_{\perp}$ by $X$.

In Figs. 1 to 3 we have plotted the ratios of the calculated $\S$ to the observed ${ }^{3}$ absorptivities, using both

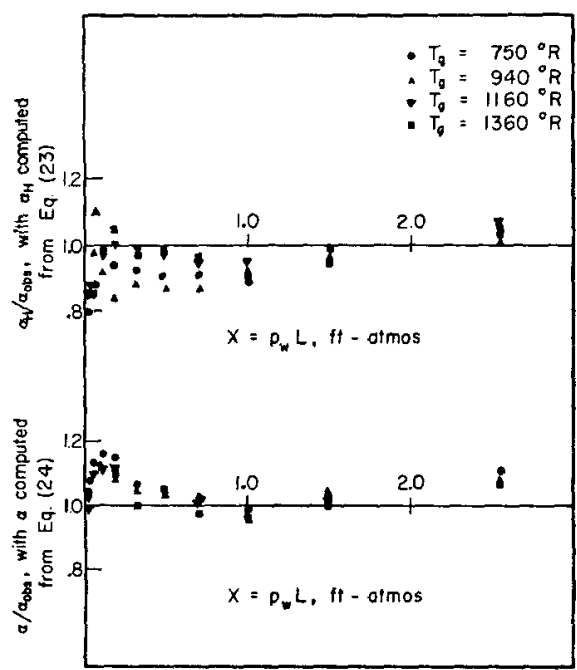

FIG. 2. The ratios $\alpha_{H} / \alpha_{\text {obs }}$ and $\alpha / \alpha_{o b \text { g }}$ for blackbody radiation at a temperature $T_{s}=1760^{\circ} \mathrm{R}$.

$\S$ For calculation of the absorptivities $\alpha_{H}$ and $\alpha$, values of $E(T, X)$ tabulated by Hottel $^{3}$ were used. The hemispherical emissivity $E(T, X)$ has been plotted as a function of the temperature $T$ and the optical density $X=P_{w} L$ for a total pressure $P_{T}$
Eqs. (23) and (24), for various values of the gas temperature $T_{g}$ and blackbody temperature $T_{s}$. Reference to Figs. 1 to 3 shows that Eqs. (23) and (24) represent the experimental data about equally well. In this connection it is of interest to note that the measured absorptivities were obtained at a total pressure of one atmosphere for partial pressures of water vapor varying from zero to one atmosphere. Since Eq. (24) was derived for overlapping rotational lines, it is not expected to apply at very low pressures and optical densities. It is apparent from Figs. 1 to 3 that significant deviations from Eq. (24) occur only at the lowest optical densities, a conclusion which is evidently in accord with expectations.

\section{B. Absorptivities of Carbon Dioxide Vapor for Blackbody Radiation}

Using measured values of the absorptivity of carbon dioxide vapor for blackbody radiation, Hottel and Mangelsdorf ${ }^{3}$ obtained the following empirical formula for the absorptivity

$$
\alpha_{H}=\left(\frac{T_{g}}{T_{s}}\right)^{0.65} E\left[T_{s}, X\left(T_{s} / T_{g}\right)\right] .
$$

For the absorption of blackbody radiation by carbon dioxide, Eq. (24) does not yield a satisfactory correlation of observed results. The reason for this discrepancy becomes apparent if it is noted that the effective bandwidth estimates obtained in Sec. II are invalid for carbon dioxide because of the presence of the exceedingly strong $\nu_{3}$ fundamental. In this case the tails of intense rotational lines located near the band center

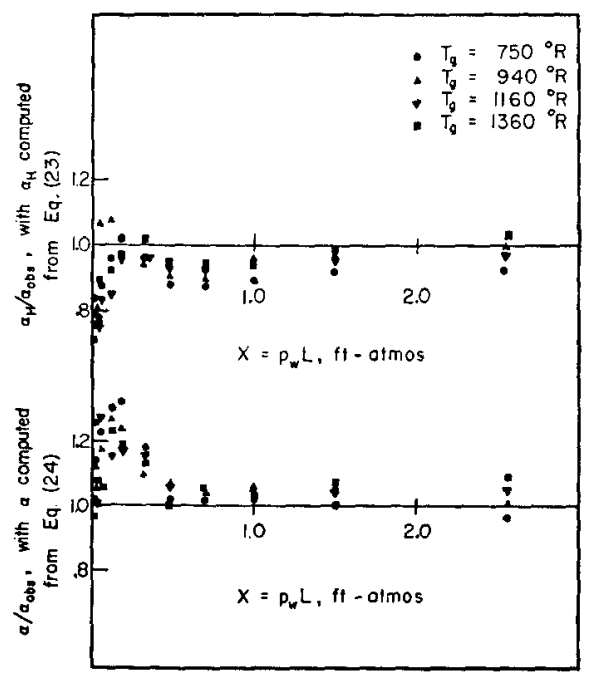

FIg. 3. The ratios $\alpha_{H} / \alpha_{o b s}$ and $\alpha / \alpha_{o b s}$ for blackbody radiation at a temperature $T_{s}=2500^{\circ} \mathrm{R}$.

of one atmosphere in the limiting case in which the water vapor partial pressure $P_{w}$ becomes negligibly small. For values of $P_{w}$ and $P_{r}$ different from 0 and 1 , respectively, an empirical correction factor $C_{w}$ must be used; this depends on $P_{T}+P_{w}$ and on $P_{w c} L^{3}$ 
make appreciable contributions at wavelengths outside of the "effective band width." Limiting conditions for failure of the theoretical band limit estimates are easily derived from this physical picture and are considered in greater detail in the Appendix.

\section{GENERAL CONCLUSIONS}

In the present paper we have used the concept of effective widths for vibration-rotation bands with average absorption coefficients to obtain an analytical expression for the radiative emissivities of diatomic molecules. The simple formulas agree with earlier numerical calculations which have been shown previously to be in accord with available empirical data.

Using the same physical model, we have derived also analytical expressions for the isothermal molecular absorptivities referring to radiation emitted from a blackbody. In accord with expectations, our formula for $\mathrm{H}_{2} \mathrm{O}$ provides a good correlation for absorptivities determined experimentally by $\mathrm{H}$. C. Hottel. On the other hand, the theoretical absorptivity results do not agree with the measured values for $\mathrm{CO}_{2}$ because the effective band width is not well defined for this molecule. This failure of the model, which we have used in the past with some measure of success in order to account for the fundamental parameters required in radiant heat transfer calculations, has prompted us to carry out a careful scrutiny of its limits of applicability. These studies are described in detail in the Appendix.

\section{APPENDIX: LIMITS OF VALIDITY OF THE EFFECTIVE BAND-WIDTH APPROXIMATION"}

The effective band-width approximation described in Sec. III fails to apply both at very low and at very high pressures and optical densities. At low pressures and optical densities, the rotational fine structure is insufficiently smeared out and the notion of an average absorption coefficient for the entire vibration-rotation band is no longer useful. On the other hand, at high pressures and optical densities, the intense rotational lines near the band center make appreciable contributions at wavelengths outside the "effective band width." In the following discussion we shall restrict our attention to the large optical density limit of the approximate calculations.

Many detailed theoretical and experimental studies of spectral line shapes have appeared in the recent literature. ${ }^{6}$ It is known that, even for impact broadening, the spectral absorption coefficients $P_{j \rightarrow j+1}(\omega)$ and

\footnotetext{
"By A. Thomson.

- See, for example, H. A. Lorentz, Proc. Akad. Sci. Amsterdam 8,59 (1906); W. Lenz, Z. Physik 80, 423 (1933); V. F. Weisskopf, Physik. Z. 34, 1 (1933); E. Lindholm, Arkiv. Mat. Astron. Fysik. 32, 17 (1945); J. H. Van Vleck and V. F. Weisskopf, Revs. Modern Phys. 17, 227 (1945); J. H. Van Vleck and H. Margenau, Phys. Rev. 76, 1211 (1949); P. W. Anderson, Phys. Rev. 76, 647 (1949); H. Margenau, Phys. Rev. 82, 156 (1951); S. Bloom and H. Margenau, Phys. Rev. 90, 791 (1953); R. E. Mayerott and H. Margenau, Phys. Rev. 99, 1851 (1955).
}

$P_{j \rightarrow j-1}(\omega)$ of the $j$ th rotational lines in the $P$ and $R$ branches, respectively, are complicated functions of the wave number $\omega .^{6}$ However, for the present purposes it is sufficient to obtain an approximate estimate of the spectral absorption coefficient near the band limit by using the Lorentz distribution

$$
P_{j \rightarrow j \pm 1}(\omega)=\frac{\left(S_{j \rightarrow j \pm 1}\right)(b / \pi)}{\left(\omega-\omega_{j \rightarrow j \pm 1}^{0}\right)^{2}+b^{2}},
$$

where $S_{j \rightarrow j+1}$ and $S_{j \rightarrow j-1}$ are the integrated intensities for the lines centered at $\omega_{j \rightarrow j+1}^{0}$ and $\omega^{0}{ }_{j \rightarrow j-1}$, in the $P$ and $R$ branches, respectively, and $b$ equals the collision (semi-) half-width, which is assumed to be uniform for the entire vibration-rotation band.

If the anharmonicity terms in the energy expression are neglected, then the spectral absorption coefficient at a wave number $\omega$ for the rotational transition $j \rightarrow j-1$ is, for large values of $j$,

$$
P_{j \rightarrow j-1}(\omega) \simeq \frac{\alpha \gamma u_{e} j\left[\exp \left(-\gamma u_{e} j^{2}\right)\right](b / \pi)}{\left(\omega-\omega^{0}{ }_{j \rightarrow j-1}\right)^{2}+b^{2}}
$$

where the line strength $S_{j \rightarrow j-1}$ has been approximated by

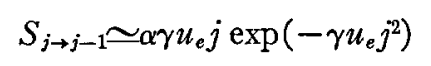

with $\alpha$ representing the integrated intensity for the entire vibration-rotation band. Similarly

$$
P_{j \rightarrow j+1}=\frac{\alpha \gamma u_{e} j\left[\exp \left(-\gamma u_{e} j^{2}\right)\right](b / \pi)}{\left(\omega-\omega^{0}{ }_{j \rightarrow j+1}\right)^{2}+b^{2}}
$$

represents the spectral absorption coefficient for the transition $j \rightarrow j+1$ whose line strength is also

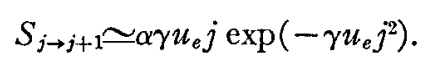

Evidently

$$
\sum_{j=0}^{\infty}\left(S_{j \rightarrow j-1}+S_{j \rightarrow j+1}\right)=\alpha .
$$

Let the wave number at the center of the vibrationrotation band, corresponding to the (forbidden) transition $j=0 \leftrightarrow j=0$, be identified by $\omega_{0}$. If the centers of the rotational lines are uniformly spaced with a wavenumber difference $q$ then

and

$$
\left(\omega-\omega_{j \rightarrow j-1}^{0}\right)^{2}=\left(\omega-\omega_{0}-q j\right)^{2}
$$

$$
\left(\omega-\omega_{j \rightarrow j+1}^{0}\right)^{2}=\left(\omega-\omega_{0}+q j\right)^{2} .
$$

The total spectral absorption coefficient at the wave number $\omega$ is

$$
P(\omega)=\sum_{j=0}^{\infty}\left(P_{j \rightarrow j-1}(\omega)+P_{j \rightarrow j+1}(\omega)\right)
$$

or, explicitly, 


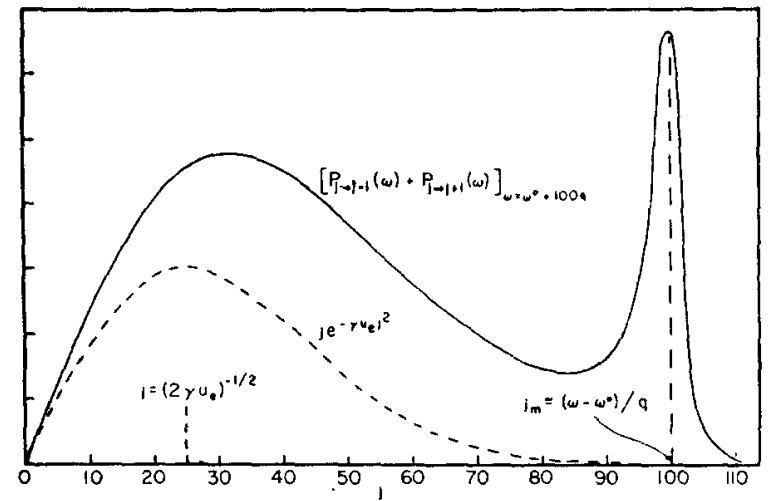

FIG. 4. The quantity $P_{j \rightarrow i-1}(\omega)+P_{j \rightarrow i+1}(\omega)$ evaluated at $\omega=\omega_{0}+100 q$ as a function of $j$ for $b / q=2.5$ and $\left(2 \gamma u_{e}\right)^{-1}=25$. The dashed curve represents $j \exp \left(-\gamma u_{\mathrm{e}} j^{2}\right)$.

$$
\begin{aligned}
P(\omega)= & \alpha \gamma u_{e}\left(\frac{b}{\pi}\right) \sum_{j=0}^{\infty} j\left[\exp \left(-\gamma u_{e} j^{2}\right)\right] \\
& \times\left[\frac{1}{\left(\omega-\omega_{0}-q j\right)^{2}+b^{2}}+\frac{1}{\left(\omega-\omega_{0}+q j\right)^{2}+b^{2}}\right]
\end{aligned}
$$

The quantity $\left(P_{j \rightarrow j-1}(\omega)+P_{j \rightarrow j+1}(\omega)\right.$ has been evaluated numerically and is plotted in Fig. 4 as a function of $j$ for $\omega=\omega_{0}+100 q, b / q=2.5$, and $\left(2 \gamma u_{e}\right)^{-1}=25$. These values apply approximately for the $\nu_{3}$ fundamental of $\mathrm{CO}_{2}$ at a temperature of $820^{\circ} \mathrm{K}$ and a pressure of 31 atmos.

For an approximate analytic evaluation of $P(\omega)$ it is convenient to separate the sum appearing in Eq. (A5) into three distinct parts which we denote by $P^{\prime}(\omega)$, $P^{\prime \prime}(\omega)$, and $P^{\prime \prime \prime}(\omega)$, such that

$$
P(\omega)=P^{\prime}(\omega)+P^{\prime \prime}(\omega)+P^{\prime \prime \prime}(\omega) .
$$

Here $P^{\prime}(\omega)$ represents the contribution to the sum in Eq. (A5) for which $0 \leqslant j \leqslant j_{m}-Q-1, P^{\prime \prime}(\omega)$ is the part for which $j_{m}-Q \leqslant j \leqslant j_{m}+Q, P^{\prime \prime \prime}(\omega)$ equals the part for $j_{m}+Q+1 \leqslant j<\infty, Q$ is an integer (which will be specified presently) much larger than $b / q$, and $j_{m}$ is the largest integer less than $\left(\omega-\omega_{0}\right) / q$. It is apparent that we have chosen $\omega$ to lie in the $P$ branch since $\omega>\omega_{0}$.

In the following Secs. A, B, and C, $P^{\prime}(\omega), P^{\prime \prime}(\omega)$, and $P^{\prime \prime \prime}(\omega)$ will be evaluated, respectively, at a wave number $\omega$ well removed from the band center, i.e., at $\omega-\omega_{0}$ appreciably larger than $2 q /\left(2 \gamma u_{e}\right)^{\frac{q}{2}}$.

\section{A. Evaluation of $P^{\prime}(\omega)$}

$P^{\prime}(\omega)$ is given by the expression

$$
\begin{aligned}
P^{\prime}(\omega)= & \alpha \gamma u_{e}\left(\frac{b}{\pi}\right)^{j_{m}-Q} \sum_{j=0}^{-1}\left[\exp \left(-\gamma u_{e} j^{2}\right)\right] \\
& \times\left[\frac{1}{\left(\omega-\omega_{0}-q j\right)^{2}+b^{2}}+\frac{1}{\left(\omega-\omega_{0}+q j\right)^{2}+b^{2}}\right] .
\end{aligned}
$$

For $Q^{2} \gg b^{2} / q^{2}$ the term $b^{2} / q^{2}$ is always negligibly small compared with $\left(\left(\omega-\omega_{0}\right) / q-j\right)^{2}$ or $\left(\left(\omega-\omega_{0}\right) / q+j\right)^{2}$ and Eq. (A6) may be approximated by

$$
\begin{aligned}
& P^{\prime}(\omega) \simeq \frac{\alpha \gamma u_{\mathrm{e}} b}{q^{2} \pi} \int_{0}^{j_{m}-Q-1} j\left[\exp \left(-\gamma u_{e} j^{2}\right)\right] \\
& \times\left\{\frac{1}{\left(\frac{\omega-\omega_{0}}{q}-j\right)^{2}}+\frac{1}{\left(\frac{\omega-\omega_{0}}{q}+j\right)^{2}}\right\} d j .
\end{aligned}
$$

Since $\left(\omega-\omega_{0}\right) / q>j_{m}-Q-\frac{1}{2}$, the integral in Eq. (A7) may be evaluated by expanding the term in parentheses in a Taylor series about $j=0$. The result is

$$
\begin{array}{r}
P^{\prime}(\omega)=\frac{\alpha b}{\pi\left(\omega-\omega_{0}\right)^{2}} \sum_{\nu=0}^{\infty}(2 \nu+1)-\frac{\nu !}{y^{\nu}}\left[1-e^{-X_{m}} \sum_{p=0}^{\nu} \frac{X_{m}^{p}}{p !}\right] \\
\equiv \frac{\alpha b}{\pi\left(\omega-\omega_{0}\right)^{2}} \eta
\end{array}
$$

where we have introduced the notation

$$
y=\gamma u_{e}\left(\omega-\omega_{0}\right)^{2} / q^{2}
$$

and

$$
X_{m}=\gamma u_{e}\left(j_{m}-Q-\frac{1}{2}\right)^{2} .
$$

For representative values of $y$ and $X_{m}$ it turns out that a considerable number of terms is required for the evaluation of $P^{\prime}(\omega)$ according to Eq. (A8). For this reason it may be preferable to determine $P^{\prime}(\omega)$ from Eq. (A7) by a graphical integration.

\section{B. Evaluation of $P^{\prime \prime}(\omega)$}

The method for evaluating $P^{\prime \prime}(\omega)$ depends on whether or not the rotational fine structure is smeared out. As will appear in the analysis, for values of $b / q \leqslant 0.5$ the treatment with allowance for fine structure is appropriate. For larger values of $b / q$ the analysis given in Sec. B(2) applies.

\section{Appreciable Rotational Fine Structure $(b / q \leqslant 0.5)$}

From Eq. (A5) and the definition of $P^{\prime \prime}(\omega)$ it is apparent that

$$
\begin{aligned}
P^{\prime \prime}(\omega)=P^{\prime \prime}{ }_{1}(\omega)+P^{\prime \prime}{ }_{2}(\omega) & \\
=\alpha \gamma u_{e}\left(\frac{b}{\pi}\right) & \left\{\sum_{j_{m}-Q}^{j_{m}+Q} \frac{j \exp \left(-\gamma u_{e} j^{2}\right)}{\left(\omega-\omega_{0}-q j\right)^{2}+b^{2}}\right. \\
& \left.+\sum_{j_{m}-Q}^{j_{m}+Q} \frac{j \exp \left(-\gamma u_{e} j^{2}\right)}{\left(\omega-\omega_{0}+q j\right)^{2}+b^{2}}\right\} .
\end{aligned}
$$

The value of $Q$ is always chosen sufficiently small to permit the approximation 


$$
\begin{aligned}
& P_{2}^{\prime \prime}(\omega)=\alpha \gamma u_{e}\left(\begin{array}{c}
b \\
-
\end{array}\right) \frac{1}{\left(\omega-\omega_{0}+q j_{m}\right)^{2}} \\
& \times \int_{j_{m}-Q}^{j_{m}+Q} j\left[\exp \left(-\gamma u_{e} j^{2}\right)\right] d j \\
& =\frac{\alpha b}{\pi} \frac{\exp \left[-\gamma u_{e}\left(j_{m}^{2}+Q^{2}\right)\right]}{\left(\omega-\omega_{0}+q j_{m}\right)^{2}} \sinh \left(2 \gamma u_{e} j_{m} Q\right) .
\end{aligned}
$$

For $Q \ll j_{m}, P_{2}^{\prime \prime}(\omega)$ is found to be very small, i.e., the contribution of $R$-branch lines at a particular wave number of the $P$ branch is negligibly small.

For sufficiently small values of $Q, P^{\prime \prime}{ }_{1}(\omega)$ may be approximated by

$$
\begin{aligned}
P^{\prime \prime}{ }_{1}(\omega)=\frac{\alpha \gamma u_{e} b}{\pi q^{2}} j_{m}\left[\exp \left(-\gamma u_{e} j_{m}{ }^{2}\right)\right] \\
\times \sum_{j=j_{m}-Q}^{j_{m}+Q}\left[\frac{1}{\left(\frac{\omega-\omega_{0}}{q}-j\right)^{2}+\left(\frac{b}{-}\right)^{2}}\right],
\end{aligned}
$$

where $j \exp \left(-\gamma u_{e} j^{2}\right)$ has been replaced by $j_{m} \exp \left(-\gamma u_{e} j_{m}^{2}\right)$, an approximation which should be fairly good in the present case since the principal contribution to $P^{\prime \prime}{ }_{1}(\omega)$, which is associated with the line identified by the quantum number $j_{m}$, has thereby been evaluated correctly. Owing to the existence of appreciable rotational fine structure, it is not permissible to replace the sum in Eq. (A13) by an integral. However, since $Q^{2} \gg b^{2} / q^{2}$, the sum appearing in Eq. (A13) may be expressed by the following relation :

$$
\begin{gathered}
\sum_{j_{m}-Q}^{j_{m}+Q}\left[\frac{1}{\left(\frac{\omega-\omega_{0}}{q}-j\right)^{2}+\frac{b^{2}}{q^{2}}}\right] \simeq \sum_{-\infty}^{\infty}\left[\frac{1}{\left(\frac{\omega-\omega_{0}}{q}-j\right)^{2}+\frac{b^{2}}{q^{2}}}\right] \\
\left.-\int_{\left(j_{m}+Q+\frac{1}{2}\right)}^{\infty}-\frac{d j}{q-\omega_{0}}\right)^{2}-\int_{-\infty}^{i_{m}-Q-\frac{1}{2}} \frac{d j}{\left(\frac{\omega-\omega_{0}}{q}-j\right)^{2}} \\
=\sum_{-\infty}^{\infty}\left[\frac{1}{\left(\frac{\omega-\omega_{0}}{q}-j\right)^{2}+\frac{b^{2}}{q^{2}}}\right]-\frac{(\mathrm{A} 14)}{\left(Q+\frac{1}{2}\right)^{2}}
\end{gathered}
$$

where $\left[j_{m}-\left(\omega-\omega_{0} / q\right)\right]^{2}$ has been neglected compared to $\left(Q+\frac{1}{2}\right)^{2}$. Using an identity valid for $B$ not an integer, viz., 7

$$
\frac{\pi}{z \sin \pi B \sin \pi(z-B)}=\sum_{-\infty}^{\infty} \frac{1}{(B+j)(z-B-j)},
$$

with $z=2 i b / q$ and $B=\left(\omega-\omega_{0}+i b\right) / q\left[\right.$ where $\left.i=(-1)^{\frac{1}{2}}\right]$, Eq. (A13) becomes

${ }^{7}$ E. T. Copson, Theory of Functions of a Complex Variable (Oxford University Press, Oxford, 1944), p. 157, Example 40.

$$
\begin{aligned}
P_{1}^{\prime \prime}(\omega)=\frac{\alpha \gamma u_{e} j_{m} \exp \left(-\gamma u_{e} j_{m}{ }^{2}\right)}{q} \\
\times\left[\frac{\tanh (2 \pi b / q)}{1-\frac{\cos 2 \pi\left(\frac{\omega-\omega_{0}}{q}\right)}{\cosh (2 \pi b / q)}}-\frac{2 b / \pi q}{\left(Q+\frac{1}{2}\right)}\right] .
\end{aligned}
$$

\section{Rotational Fine Structure Smeared Out $(b / q \geqslant 0.5)$}

For $b / q \geqslant 0.5$ the quantity $P^{\prime \prime}{ }_{2}(\omega)$ is still given in good approximation by Eq. (A12); it is again negligibly small. The second contribution to $P^{\prime \prime}(\omega)$ may now be written as

$$
P^{\prime \prime}{ }_{1}(\omega) \simeq \frac{\alpha \gamma u_{e} b}{\pi} \int_{j_{m}-Q-\frac{1}{2}}^{j_{m}+Q+\frac{1}{2}} \frac{j \exp \left(-\gamma u_{e} j^{2}\right)}{\left(\omega-\omega_{0}-q j\right)^{2}+b^{2}} d j,
$$

because, for the larger values of $b / q$, the rotational fine structure is smeared out and, therefore, the sum may be approximated by an integral. A first estimate for $P^{\prime \prime}{ }_{1}(\omega)$ is then obtained by replacing the numerator in the integral by the constant value $j_{m} \exp \left(-\gamma u_{e} j_{m}{ }^{2}\right)$. Thus

$$
\begin{aligned}
& P_{1}^{\prime \prime}(\omega) \simeq \frac{\alpha \gamma u_{e}}{\pi q} j_{m}\left[\exp \left(-\gamma u_{e} j_{m}{ }^{2}\right)\right] \\
& \quad \times\left\{\tan ^{-1}\left[\frac{\left(j_{m}+Q+\frac{1}{2}\right) q-\left(\omega-\omega_{0}\right)}{b}\right]\right. \\
& \left.\quad-\tan ^{-1}\left[\frac{\left(j_{m}-Q-\frac{1}{2}\right) q-\left(\omega-\omega_{0}\right)}{b}\right]\right\} ;
\end{aligned}
$$

in particular, at the center of the $j_{m}$ th line where $\omega-\omega_{0}=j_{m} q$,

$$
\begin{aligned}
P^{\prime \prime}{ }_{1}\left(\omega=\omega_{0}+j_{m} q\right) \simeq \frac{2 \alpha \gamma u_{e}}{\pi q} j_{m} & {\left[\exp \left(-\gamma u_{e} j_{m}{ }^{2}\right)\right] } \\
& \times \tan ^{-1}\left[\left(Q+\frac{1}{2}\right) q / b\right] .
\end{aligned}
$$

since $\tan ^{-1}\left[\left(Q+\frac{1}{2}\right) q / b\right]=-\tan ^{-1}\left[-\left(Q+\frac{1}{2}\right) q / b\right]$.

A more satisfactory evaluation for the integral of Eq. (A17) may be obtained by expanding the term $j \exp \left(-\gamma u_{e} j^{2}\right)$ in a Taylor series around $j=j_{m}$. We find

$$
\begin{aligned}
& j \exp \left(-\gamma u_{e} j^{2}\right) \\
& =\exp \left(-\gamma u_{e} j_{m}{ }^{2}\right)\left\{j_{m}-\left(2 \gamma u_{e} j_{m}{ }^{2}-1\right)\left(j-j_{m}\right)\right. \\
& +\left[2 \gamma u_{e} j_{m}\left(2 \gamma u_{e} j_{m}{ }^{2}-3\right)\right] \frac{\left(j-j_{m}\right)^{2}}{2} \\
& +\left[-3\left(2 \gamma u_{e}\right)+6\left(2 \gamma u_{e}\right)^{2} j_{m}{ }^{2}-\left(2 \gamma u_{e}\right)^{3} j_{m}{ }^{4}\right] \frac{\left(j-j_{m}\right)^{3}}{6} \\
& +\left[15\left(2 \gamma u_{e}\right)^{2} j_{m}-10\left(2 \gamma u_{e}\right)^{3} j_{m}{ }^{3}+\left(2 \gamma u_{e}\right)^{4} j_{m}{ }^{5}\right] \\
& \left.\times \frac{\left(j-j_{m}\right)^{4}}{24}-\cdots\right\} \quad(\text { A } 20)
\end{aligned}
$$


Since $P^{\prime \prime}{ }_{1}(\omega)$ varies smoothly with $\omega$ for sufficiently large values of $b / q$, it is sufficient to evaluate $P^{\prime \prime}{ }_{1}(\omega)$ at $\omega=\omega_{0}+j_{m} q$ and to connect the resulting discrete points in order to obtain the curve of $P^{\prime \prime}{ }_{1}(\omega)$ as a function of $\omega$. Neglecting the quartic and higher powers of $j-j_{m}$ in Eq. (A20), it is now found from Eqs. (A17) and $(\mathrm{A} 20)$ that

$$
\begin{array}{r}
P_{1}^{\prime \prime}\left(\omega=\omega_{0}+j_{m} q\right)=\frac{\alpha \gamma u_{e} j_{m} \exp \left(-\gamma u_{e} j_{m}{ }^{2}\right)}{q} \\
\times\left\{\frac{2}{\pi} \tan ^{-1}\left[\left(Q+\frac{1}{2}\right) q / b\right]+\frac{2 \gamma u_{e}}{\pi} \frac{b^{2}}{q^{2}}\left(2 \gamma u_{e} j_{m}{ }^{2}-3\right)\right. \\
\left.\times\left\{\left(Q+\frac{1}{2}\right) q / b-\tan ^{-1}\left[\left(Q+\frac{1}{2}\right) q / b\right]\right\}\right\} .
\end{array}
$$

\section{Evaluation of $P^{\prime \prime \prime}(\omega)$}

For the sake of simplicity we restrict the following evaluation to $\omega=\omega_{0}+j_{m} / q$. Since $Q^{2} \gg b^{2} / q^{2}$ we find from

$$
\begin{aligned}
P^{\prime \prime \prime}\left(\omega_{0}+j_{m} / q\right)= & \frac{\alpha \gamma u_{e} b}{\pi q^{2}} \sum_{j=j_{m}+Q+1}^{\infty} j\left[\exp \left(-\gamma u_{e} j^{2}\right)\right] \\
& \times\left[\frac{1}{\left(j-j_{m}\right)^{2}+(b / q)^{2}}+\frac{1}{\left(j+j_{m}\right)^{2}+(b / q)^{2}}\right]
\end{aligned}
$$

the following relation for (overlapping) rotational lines:

$$
\begin{array}{r}
P^{\prime \prime \prime}\left(\omega_{0}+j_{m} / q\right) \simeq \frac{\alpha \gamma u_{e} b}{\pi q^{2}} \int_{j m+Q+\frac{1}{2}}^{\infty} j\left[\exp \left(-\gamma u_{e} j^{2}\right)\right] \\
\times\left[\frac{1}{\left(j-j_{m}\right)^{2}}+\frac{1}{\left(j+j_{m}\right)^{2}}\right] d j .
\end{array}
$$

The integral in Eq. (A22) can be evaluated conveniently by expanding the terms $j \exp \left(-\gamma u_{e} j^{2}\right)$ and $\left(j-j_{m}\right)^{-2}$ in power series around the lower limit of integration $j=j_{m}+Q+\frac{1}{2}$. In this manner it is easily shown that

$$
\begin{array}{r}
P^{\prime \prime \prime}\left(\omega_{0}+j_{m} / q\right)<\frac{\alpha \gamma u_{e} b}{\pi q^{2}}\left[\exp \left(-\gamma u_{e}\left(j_{m}+Q+\frac{1}{2}\right)^{2}\right)\right] \\
\quad \times\left(j_{m}+Q+\frac{1}{2}\right)\left[\frac{1}{\left(Q+\frac{1}{2}\right)}+\frac{1}{\left(2 j_{m}+Q+\frac{1}{2}\right)}\right] .
\end{array}
$$

However, according to Eq. (A8),

$$
P^{\prime}\left(\omega_{0}+j_{m} / q\right)=\frac{\alpha b}{\pi q^{2} j_{m}^{2}} \eta,
$$

where $\eta=1.7$ for the following typical values: $j_{m} / j^{*}=4$, $j_{m}=100, y=8, Q=5, \gamma u_{e}=1 / 2\left(j^{*}\right)^{2}=1 / 1250, x_{m} \simeq 7$.
For the given numerical values we find from Eqs. (A22) and (A23) that

$$
\begin{aligned}
& \frac{P^{\prime \prime \prime}\left(\omega_{0}+j_{m} / q\right)}{P^{\prime}\left(\omega_{0}+j_{m} / q\right)}<\frac{1}{2 \eta}\left(\frac{j_{m}}{j^{*}}\right)^{2}\left\{\exp \left[-\frac{1}{2}\left(\frac{j_{m}+Q+\frac{1}{2}}{j^{*}}\right)^{2}\right]\right\} \\
& \times\left(j_{m}+Q+\frac{1}{2}\right)\left[\frac{1}{\left(Q+\frac{1}{2}\right)}+\frac{1}{\left(2 j_{m}+Q+\frac{1}{2}\right)}\right]=0.012
\end{aligned}
$$

Thus it has been demonstrated that the contribution of $P^{\prime \prime \prime}(\omega)$ to $P(\omega)$ is negligibly small.

\section{Total Spectral Absorption Coefficient}

The total absorption coefficient has been shown to be approximately

$$
P(\omega)=P^{\prime}(\omega)+P^{\prime \prime}{ }_{1}(\omega) .
$$

According to Eq. (A8),

$$
P^{\prime}(\omega)=\frac{\alpha b}{\pi\left(\omega-\omega_{0}\right)^{2}} \eta,
$$

where the given expression for $P^{\prime}(\omega)$ applies for all values of $b / q$ provided $Q$ is chosen in accord with the condition $Q^{2} \gg b^{2} / q^{2}$. The quantity $P^{\prime \prime}{ }_{1}(\omega)$ is determined by Eq. (A16) for $b / q \leqslant 0.5$; it is given by Eq. (A21) for $b / q \geqslant 0.5$. We note that for $b / q \gg 1$ the first two terms in Eq. (A16) become identical with the value of $(2 / \pi) \tan ^{-1}\left[(q / b)\left(Q+\frac{1}{2}\right)\right]$. Therefore the sum of the two terms appearing in the braces of Eq. (A16), when added to the second term of Eq. (A21), is a valid representation for $b / q \gg 1$. On the other hand, for small values of $b / q$ the two terms in the braces of $\mathrm{Eq}$. (A16) reduce to roughly $\pi(b / q)$ whereas the correction term to the arctangent in Eq. (A21) is about two orders of magnitude smaller for $Q=5, j_{m}=100, j^{*}=\left(2 \gamma u_{e}\right)^{-\frac{1}{2}}$ $=25$. Hence, for all values of $b / q$, we have the following approximate representation for $P^{\prime \prime}{ }_{1}(\omega)$ :

$$
\begin{aligned}
P^{\prime \prime}{ }_{1}(\omega)=\frac{\alpha \gamma u_{e} j_{m}}{\exp \left(-\gamma u_{e} j_{m}{ }^{2}\right)} & \\
& \times\left\{\left\{1-\frac{\cos \left[2 \pi\left(\omega-\omega_{0}\right) / q\right]}{\cosh (2 \pi b / q)}\right\}^{-1}\right. \\
& \times \tanh \left(\frac{2 \pi b}{q}\right)-\frac{(2 b / \pi q)}{\left(Q+\frac{1}{2}\right)} \\
& +\frac{2 \gamma u_{e} b^{2}}{\pi}\left(2 \gamma u_{e} j_{m}{ }^{2}-3\right) \\
& \left.\times\left\{\frac{q}{b}\left(Q+\frac{1}{2}\right)-\tan ^{-1}\left[\frac{q}{b}\left(Q+\frac{1}{2}\right)\right]\right\}\right\} .
\end{aligned}
$$


TABL.E II. Relevant spectroscopic constants for $\mathrm{CO}, \mathrm{HCl}, \mathrm{CO}_{2}$, and $\mathrm{H}_{2} \mathrm{O}$.

\begin{tabular}{|c|c|c|c|}
\hline Molecule & $\left(\mathrm{cm}^{-2}\right.$ atmos $\left.^{-1}\right)$ & $\underset{\left(\mathrm{cm}^{-1}\right)}{q}$ & $\begin{array}{c}b_{F^{1}} \\
\left(\mathrm{~cm}^{-1}\right)\end{array}$ \\
\hline $\mathrm{CO}$ (fundamental) & $237(300 / T)^{\mathrm{a}}$ & 3.86 & $0.1 p(T / 300)^{-\frac{1}{2}}$ \\
\hline HCl(fundamental) & $160(300 / T)^{\mathrm{b}}$ & 21.2 & $0.1 p(T / 300)^{-1}$ \\
\hline $\mathrm{CO}_{2}\left(\nu_{\mathrm{s}}\right.$ fundamental $)$ & $2700(300 / T)$ & 0.791 & $0.1 p(T / 300)^{-1}$ \\
\hline $\mathrm{H}_{2} \mathrm{O}$ (hypothetical band) & $<1000(300 / T)^{d}$ & $\sim 10$ & $0.1 p(T / 300)^{-\frac{1}{2}}$ \\
\hline
\end{tabular}

aS. S. Penner and D. Weber, J. Chem. Phys, 19, 807 (1951).

hS. S. Penner and D. Weber, J. Chem. Phys. 21, 649 (1953)

- Weber, Holm, and Penner, J. Chem. Phys. 20, 1820 (1952)

A Assumed value.

- Representative experimental data suggest that $b_{P^{\prime}} \sim 0.10 \mathrm{~cm}^{-1}$ at 1 atmos and $300^{\circ} \mathrm{K}$. See, for example, D. Weber and S. S. Penner, J. Chem. Phys. 21, 1503 (1953).

\section{E. Large Optical Density Limit for the Validity of the "Effective Band-Width Approximation"}

The limiting value of the optical density $X_{u}$, for which our effective band-width estimate applies, may be defined by

$$
X_{u}=0.2 / P^{\prime}\left(\omega_{0}+\Delta \omega / 2\right)
$$

since $P^{\prime}\left(\omega_{0}+\Delta \omega / 2\right)$ is a slowly varying function of $\Delta \omega$. Evidently $P^{\prime}\left(\omega_{0}+\Delta \omega / 2\right)$ is the contribution to $P(\omega)$ of the tails of the intense rotational lines at the limit of the effective band width. For $\eta=1.7$, Eq. (A26) becomes

$$
X_{u} \simeq 0.1 \pi(\Delta \omega)^{2} / 4 \alpha b .
$$

Relevant spectroscopic data for $\mathrm{CO}, \mathrm{HCl}, \mathrm{CO}_{2}$, and $\mathrm{H}_{2} \mathrm{O}$ are given in Table II for the most intense vibrationrotation bands. In Table III the values of $X_{u}$ are listed for various temperatures and pressures.

\begin{tabular}{|c|c|c|c|c|}
\hline Molecule & $\stackrel{T}{\left({ }^{T} \mathbf{K}\right)}$ & $\underset{\left(\mathrm{cm}^{-1}\right)}{\Delta \omega}$ & $\begin{array}{c}\text { for } b / q=0.1 \\
X_{u} \\
\text { (cm atmos) }\end{array}$ & $\begin{array}{c}X_{u} \text { at } \\
p=1 \text { atmos } \\
\text { (cm atmos) }\end{array}$ \\
\hline $\mathrm{CO}$ (fundamental) & $\begin{array}{r}300 \\
1000 \\
3000\end{array}$ & $\begin{array}{l}239 \\
436 \\
756\end{array}$ & $\begin{array}{r}74 \\
820 \\
7400\end{array}$ & $\begin{array}{r}370 \\
7700 \\
114000\end{array}$ \\
\hline $\mathrm{HCl}$ (fundamental) & $\begin{array}{r}300 \\
1000 \\
3000\end{array}$ & $\begin{array}{r}560 \\
1022 \\
1722\end{array}$ & $\begin{array}{r}35000 \\
>35000 \\
>35000\end{array}$ & $\begin{array}{r}700000 \\
>700000 \\
>700000\end{array}$ \\
\hline $\mathrm{CO}_{2}\left(\nu_{3}\right.$ fundamental $)$ & $\begin{array}{r}300 \\
1000 \\
3000\end{array}$ & $\begin{array}{l}108 \\
197 \\
342\end{array}$ & $\begin{array}{l}2.6 \\
30 \\
260\end{array}$ & $\begin{array}{r}2.1 \\
45 \\
660\end{array}$ \\
\hline $\begin{array}{l}\mathrm{H}_{2} \mathrm{O} \text { (hypothetical } \\
\text { band for } \alpha_{F}<1000 \\
\mathrm{~cm}^{-2} \text { atmos } \\
300^{-1} \mathrm{~K} \text { ) at }\end{array}$ & $\begin{array}{r}300 \\
1000 \\
3000\end{array}$ & $\begin{array}{r}400 \\
720 \\
1250\end{array}$ & $\begin{array}{r}>1200 \\
>14000 \\
>120000\end{array}$ & $\begin{array}{l}>12000 \\
>12000 \\
>12000\end{array}$ \\
\hline
\end{tabular}

'TABLE III. Optical density limits $X_{u}$ for $\mathrm{CO}, \mathrm{HCl}, \mathrm{CO}_{2}$, and $\mathrm{H}_{2} \mathrm{O}$.

\section{F. Low Optical Density Limit for the Validity of the Effective Band-Width Approximation}

The practical optical density limit $\uparrow$ for the validity of the effective band-width approximation cannot be determined, in general, without a careful study of the relative importance of different vibration-rotation bands. In general, a more profitable line of approach than a detailed study of line structure in the wings of vibration-rotation bands involves a direct comparison of engineering emissivities evaluated according to various limiting procedures. ${ }^{1,2}$

T This phrase is to be interpreted in the sense that the engineering emissivity calculations are good to perhaps $20 \%$. 